\title{
The Covering Homotopy Extension Problem for Compact Transformation Groups
}

\author{
S. M. Ageev ${ }^{* *}$ and D. Repovš $\check{2}^{\text {*** }}$ \\ 'Belarusian State University \\ ${ }^{2}$ University of Ljubljana, Slovenija \\ Received November 15, 2010; in final form, October 9, 2011
}

\begin{abstract}
It is shown that the orbit space of universal (in the sense of Palais) $G$-spaces classifies $G$-spaces. Theorems on the extension of covering homotopy for $G$-spaces and on a homotopy representation of the isovariant category ISOV are proved .
\end{abstract}

DOI: $10.1134 / \mathrm{S} 0001434612110016$

Keywords: $G$-space, covering homotopy, compact transformation group, orbit space, universal G-space in the sense of Palais, absolute (neighborhood) extensor, classifying space.

\section{INTRODUCTION}

This paper studies the isovariant category ISOV, whose objects are spaces with an action of a compact group $G$ and morphisms are isovariant maps. Historically, the objects of this category are usually treated as generalized principal $G$-bundles and its injective objects (三Isov-AE-spaces), as universal generalized principal $G$-bundles. Objects with similar properties were first constructed by Palais for compact Lie groups acting on finite-dimensional spaces with finitely many orbit types [1, Sec. 2.6.]. Afterward, this result was extended to compact metrizable groups acting on spaces with finite-dimensional orbit space [2]. In [3], the last constraints were removed, and an existence theorem for Isov-AE-spaces was proved.

Theorem 1. If $\mathbb{X}_{i}$ is an Isov-generating Equiv-AE-space for any $i \geq 1$, then

$$
\prod\left\{\mathbb{X}_{i} \mid i \geq 1\right\} \in \text { Isov-AE. }
$$

A space $\mathbb{X}$ is said to be Isov-generating if, for any metric $G$-space $\mathbb{Z}$, there exists an isovariant map $\eta: \mathbb{Z} \rightarrow \mathbb{X}$. It was proved in [4] that the countable power $\mathbb{J} \rightleftharpoons(\text { Con } \mathbb{T})^{\omega}$ of the metric cone Con $\mathbb{T}$ over the discrete union $\mathbb{T}$ of all homogeneous spaces $G / H \in G$-ANE is Isov-generating (see also [5]). Since $\mathbb{J}$ is also an Equiv-AE-space and $\mathbb{J} \cong \mathbb{J}^{\omega}$, it follows from Theorem 1 that $\mathbb{J} \in$ Isov-AE.

Each family $\mathcal{F}$ belonging to the set $\operatorname{Conj}_{G}$ of conjugate classes of closed subgroups of $G$ generates a series of equivariant homotopy invariants, including $\mathcal{F}$-classifying $G$-spaces in the sense of [6], [7] and generalized cohomology groups [8], [9], which are closely related to them, as well as fundamental classes of $G$-spaces and other equivariant homotopy invariants [10]. Theorem 1 makes it possible to prove that, on $\mathcal{F}$-classifying $G$-spaces, the additional structure of isovariant absolute extensors can be defined, which opens new possibilities for calculating homotopy invariants of the orbit spaces of $\mathcal{F}$ classifying $G$-spaces. In turn, this gives important information about the generalized cohomology of compact groups.

Theorem 1 also implies the important conclusion that the equivariant homotopy type of Equiv-ANEspaces coincides with the isovariant homotopy type of Isov-ANE-spaces. This clarifies the functorial nature of the operation of the passage to the bundle of orbits of a given type, which is not preserved by

\footnotetext{
${ }^{*}$ E-mail: ageev_sergei@yahoo.com

${ }^{* *}$ E-mail: dusan.repovs@guest. arnes.si
} 
equivariant homotopy equivalences. The circle of questions outlined above will be considered elsewhere (see, e.g., [3], [11]).

In this paper, we apply Theorem 1 to prove that the orbit space $E$ of any Isov-AE-space $\mathbb{E}$ classifies $G$-spaces in the sense of Palais. To this end, we carry over Palais' classical result on covering homotopy (see [1], [2]) to arbitrary compact groups and obtain a more general result on the extension of covering homotopy (Theorem 2). By virtue of this result, the category ISOV admits a homotopy representation, which largely reduces studying this category to considering homotopy properties of topological spaces (see Theorem 5).

\section{Statement of the Covering Homotopy Extension Problem}

Let $G$ be a compact group. Consider the following commutative square $G$-diagram $\mathcal{D}$ :

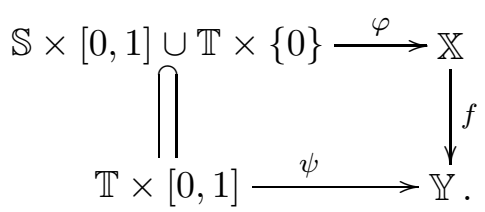

In this diagram, $\mathbb{S} \subset \mathbb{T}$ is a closed $G$-subspace and $f, \varphi$, and $\psi$ are $G$-maps. We say that the diagram $\mathcal{D}$ is admissible (weakly admissible) for the $G$-map $f$ if

(a) $\varphi$ is an isovariant map (respectively, $\varphi$ is an isovariant map and $\psi$ induces a homeomorphism $\psi: T \times[0,1] \rightarrow Y$ of the orbit spaces);

(b) $\left(f^{-1} \circ \psi\right)(G(u) \times I)$ has single orbit type $\left(G_{u}\right)$ for each $u \in T$.

Any admissible commutative diagram $\mathcal{D}$ satisfies the following conditions:

1) the orbit types of the spaces $\mathbb{X}$ and $\mathbb{T}$ are related by $\mathrm{Orb}_{\mathbb{T}} \subset \mathrm{Orb}_{\mathbb{X}}$;

2) if the map $f$ is isovariant, then so is the map $\psi$.

If $\mathcal{D}$ is an admissible diagram and $f$ is a $P$-orbit projection, then

3) the induced map $\psi_{P}: \mathbb{T} / P \times[0,1] \rightarrow \mathbb{Y}$ given by $\left(\psi_{P}\right)(P \cdot u, t)=\psi(u, t)$ is well defined, and this map is an isovariant embedding.

We say that a $G$-map $\widehat{\varphi}: \mathbb{T} \times[0,1] \rightarrow \mathbb{X}$ splits the diagram $\mathcal{D}$ if $\widehat{\varphi}$ is a lift of $\psi$ with respect to $f$ (i.e., $f \circ \widehat{\varphi}=\psi$ ) and $\widehat{\varphi}=\operatorname{ext} \varphi$. By virtue of (b), the map $\widehat{\varphi}$ is necessarily isovariant (i.e., $\varphi=\widehat{\varphi}$ on $\mathbb{S} \times[0,1] \cup \mathbb{T} \times\{0\})$.

We say that the isovariant covering homotopy extension problem is solvable (weakly solvable) for a $G$-map $f: \mathbb{X} \rightarrow \mathbb{Y}$ if any admissible $G$-diagram (any weakly admissible $G$-diagram) $\mathcal{D}$ for $f$ splits. In this case, we say that the $G$-map $f: \mathbb{X} \rightarrow \mathbb{Y}$ is a Hurewicz Isov-bundle (a weak Hurewicz Isovbundle). Clearly, any Hurewicz Isov-bundle is a weak Hurewicz Isov-bundle. The following theorem plays the key role in this paper.

Theorem 2. Any orbit projection $p: \mathbb{X} \rightarrow X$ is a Hurewicz Isov-bundle.

Now, applying the equimorphism criterion to an isovariant map $\widehat{\varphi}$ splitting a weakly admissible $G$-diagram $\mathcal{D}$ from which $\mathbb{S}$ is missing, we obtain a generalization to any compact groups of Palais' well-known theorem on the equivariant type of a space having the orbit type of the product $\mathbb{W} \times[0,1]$ (see [1]).

The proof of Theorem 2 is based on the following result, with is of independent interest.

Theorem 3. Let $\pi: G \rightarrow H$ be an epimorphism of compact groups whose kernel $P=\operatorname{Ker} \pi$ is a Lie group. Then any $P$-orbit projection $f: \mathbb{X} \rightarrow \mathbb{Y}=\mathbb{X} / P$ is a Hurewicz Isov-bundle.

We reduce Theorem 2 to Theorem 3 as follows. First, we expand the $G$-space $\mathbb{X}$ in a Lie series $\left\{\mathbb{X}_{\alpha}, \pi_{\alpha}^{\beta}\right\}$. Since $P_{\alpha} / P_{\alpha+1}$ is a compact Lie group, it follows by Theorem 3 that the bonding $P_{\alpha}^{\alpha+1}$-orbit projection $\pi_{\alpha}^{\alpha+1}: \mathbb{X}_{\alpha+1} \rightarrow \mathbb{X}_{\alpha}$ is a Hurewicz Isov-bundle for each $\alpha<\tau$. Then, arguing by transfinite induction, we prove that the limit orbit projection $\pi: \mathbb{X} \rightarrow X$ is a Hurewicz Isov-bundle as well. 


\section{Homotopy Representation of the Category ISOV}

On the orbit space $X$ of a $G$-space $\mathbb{X}$, we consider the stratification $\left\{X_{(H)} \mid H<G\right\}$ generated by the orbit types (the space $X$ itself is called an $\mathcal{S}$-space); a map $\alpha: X \rightarrow E$ of $\mathcal{S}$-spaces is said to be stratification-preserving (or, briefly, an $\mathcal{S}$-map) if

$$
\alpha\left(X_{(H)}\right) \subset E_{(H)} \quad \text { for any subgroup } \quad H<G .
$$

An $\mathcal{S}$-homotopy $H: X \times I \rightarrow E$ between stratification-preserving maps is defined in a similar way (i.e., it is required that $H\left(X_{(H)} \times I\right) \subset E_{(H)}$ for any $\left.H<G\right)$.

The fiber product of spaces $C$ and $B$ with respect to maps $C \stackrel{g}{\rightarrow} A$ and $B \stackrel{f}{\rightarrow} A$ is defined as the set

$$
\{(c, b) \mid g(c)=f(b)\} \subset C \times B,
$$

which we denote by $C{ }_{g} \times{ }_{f} B$ or, briefly, by $g^{*}(B)$ or $f^{*}(C)$. The projections $D=C_{g} \times{ }_{f} B$ onto the factors $C$ and $B$ determine maps $f^{*}: D \rightarrow C$ and $g^{*}: D \rightarrow B$. We refer to $f^{*}$ as the map parallel to $f$ and to the map $g^{*}$ as the map parallel to $g$.

An equimorphism criterion (Proposition 2) relates isovariant maps to fiber products.

Proposition 1. Let $h: \mathbb{Y} \rightarrow \mathbb{X}$ be an isovariant map, and let $\widetilde{h}: Y \rightarrow X$ be the map of orbit spaces generated by $h$. Then the $G$-map $h$ and the $G$-map $(\widetilde{h})^{*}:(\widetilde{h})^{*}(\mathbb{X}) \rightarrow \mathbb{X}$ parallel to $\widetilde{h}$ are equimorphic, i.e., there exists a G-homeomorphism $\theta:(\widetilde{h})^{*}(\mathbb{X}) \rightarrow \mathbb{Y}$ for which $h \circ \theta=(\widetilde{h})^{*}$ (thereby, $\mathbb{Y}$ is identified with the fiber product $\widetilde{h}^{*}(\mathbb{X})$ ).

Using Theorem 2 and Proposition 1, we prove that the $G$-spaces determined by homotopic $\mathcal{S}$-maps connected by an $\mathcal{S}$-homotopy are equimorphic; namely, the following theorem is valid.

Theorem 4. If $\mathcal{S}$-maps $\alpha: X \rightarrow E$ and $\beta: X \rightarrow$ E are connected by an $\mathcal{S}$-homotopy, then the fiber products

$$
\alpha^{*}(\mathbb{E})=\mathbb{E}_{\pi} \times_{\alpha} X \quad \text { and } \quad \beta^{*}(\mathbb{E})=\mathbb{E}_{\pi} \times_{\beta} X
$$

are equimorphic (here $\pi: \mathbb{E} \rightarrow E$ is the orbit projection).

If a $G$-space $\mathbb{E}$ is an Isov-AE, then the converse is also true; that is, if $\alpha^{*}(\mathbb{E}) \cong_{G} \beta^{*}(\mathbb{E})$, then the $\mathcal{S}$-maps $\alpha: X \rightarrow E$ and $\beta: X \rightarrow$ E are connected by an $\mathcal{S}$-homotopy.

Let $\mathcal{S}-$ HOMOT $_{E}$ denote the category whose objects are $\mathcal{S}$-homotopy classes $[\alpha]: X \rightarrow E$ of $\mathcal{S}$-maps and the morphism between $\mathcal{S}$-maps $[\alpha]$ and $[\beta]: Y \rightarrow E$ is the $\mathcal{S}$-homotopy class of the $\mathcal{S}$-map $h: X \rightarrow Y$ for which $\alpha=\beta \circ h$.

To each object $[\alpha]: X \rightarrow E$ of the category $\mathcal{S}$-HOMOT $E$ we assign the $G$-space coinciding with the fiber product $\mathbb{X} \rightleftharpoons \alpha^{*}(\mathbb{E})$, and to each morphism $[h]: X \rightarrow Y$ we assign the isovariant map

$$
[h]_{*}: \mathbb{X}=\alpha^{*}(\mathbb{E}) \cong_{G}\left(\alpha^{\prime}\right)^{*}(\mathbb{E}) \stackrel{h_{*}}{\longrightarrow} \beta^{*}(\mathbb{E}) \rightleftharpoons \mathbb{Y}, \quad \text { where } \quad h_{*}(x, e)=(h(x), e) .
$$

It follows readily from Theorem 4 that, under certain conditions, the covariant functor $\Phi$ thus constructed is an isomorphism between the isovariant homotopy category ISOV-HOMOT and the category $\mathcal{S}-\mathrm{HOMOT}_{E}$.

Theorem 5. If $\mathbb{E}$ is an Isov-AE-space, then

$$
\Phi: \mathcal{S}-\mathrm{HOMOT}_{E} \rightarrow \text { ISOV-HOMOT }
$$

is an equivalence of categories.

Theorem 5 can be regarded as an additional argument supporting the thesis that the isovariant category is a generalization of the category of principal $G$-bundles.

MATHEMATICAL NOTES Vol. 92 No. 62012 


\section{PRELIMINARY INFORMATION AND RESULTS}

From now on, all spaces (maps) if they do not arise as the result of certain constructions and if it is not otherwise specified, are assumed to be metric (continuous); we consider only actions of compact groups.

Below we recall the basic notions of the theory of $G$-spaces [12]. By an action of a compact group $G$ on a space $\mathbb{X}$ we mean a continuous map $\mu$ from the product $G \times \mathbb{X}$ to $\mathbb{X}$ which satisfies the conditions

$$
\mu(g, \mu(h, x))=\mu(g \cdot h, x), \quad \mu(e, x)=x \quad \text { for all } \quad x \in \mathbb{X}, \quad g, h \in G
$$

(here $e$ denotes the identity element of the group $G$ ). As a rule, instead of $\mu(g, x)$ we write $g \cdot x$ or simply $g x$. A space $\mathbb{X}$ with an action of a group $G$ is called a $G$-space. A map $f: \mathbb{X} \rightarrow \mathbb{Y}$ of $G$-spaces is said to be a $G$-map, or an equivariant map, if $f(g \cdot x)=g \cdot f(x)$ for all $x \in \mathbb{X}$ and $g \in G$.

Note that all $G$-spaces and $G$-maps form a category, which we denote by $G$-TOP or by EQUIV, if it is clear what group $G$ is considered. We freely use the symbols " $G-$ " and "Equiv-", which mean "equivariant." If "***" is a certain notion from nonequivariant topology, then " $G-* * *$ " and "Equiv$* * *$ " stand for the corresponding equivariant counterpart.

The orbit $G(x)$ of a point $x \in \mathbb{X}$ is defined as the subset $\{g \cdot x \mid g \in G\}=G \cdot x$; this subset is always closed. The natural map

$$
\pi=\pi_{\mathbb{X}}: \mathbb{X} \rightarrow X, \quad x \mapsto G(x),
$$

of the space $\mathbb{X}$ to the space $X \rightleftharpoons \mathbb{X} / G$ of the quotient partition is called the orbit projection. The space $X$ of the quotient partition endowed with the quotient topology generated by $\pi$ is called the orbit space. A subset $A$ is said to be invariant, or a $G$-subset, if $\pi^{-1} \pi(A)=G \cdot A$.

By $\operatorname{Conj}_{G}$ we denote the set of all conjugacy classes of the closed subgroups of $G$ and by $\operatorname{Orb}_{G}$, the family of all homogeneous spaces up to equimorphism. On these sets, we introduce the following partial orders:

$$
\begin{aligned}
(K) \leq(H) & \Longleftrightarrow K \text { is contained in some representative } H^{\prime} \text { of the class }(H), \\
G / K \geq G / H & \Longleftrightarrow \text { there exists an equivariant map } f: G / K \rightarrow G / H ;
\end{aligned}
$$

the bijection

$$
(H) \in \operatorname{Conj}_{G} \mapsto G / H \in \operatorname{Orb}_{G}
$$

reverses these orders. Taking into account this observation, we shall identify the sets specified above and use the same name, the set of $G$-orbit types, and the same notation $\operatorname{Orb}_{G}$ for these sets at all places where such an identification causes no ambiguity.

For each point $x \in \mathbb{X}$, the subset

$$
G_{x}=\{g \in G \mid g \cdot x=x\}
$$

is a closed subgroup in the group $G$; this subgroup is called the stabilizer of the point $x$. For any closed subgroup $H<G$, we define the following subsets of $\mathbb{X}$ :

- $\mathbb{X}^{H}=\{x \in \mathbb{X} \mid H \cdot x=x\}=\left\{x \in \mathbb{X} \mid H \subset G_{x}\right\}$ (this is the $H$-fixed point set);

- $\mathbb{X}_{H}=\left\{x \in \mathbb{X} \mid H=G_{x}\right\}$;

- $\mathbb{X}_{(H)}=\left\{x \in \mathbb{X} \mid H\right.$ is conjugate to $\left.G_{x}\right\}$ (this is the bundle of orbits of type $(H)$ ).

By Orb $\mathbb{X}$ we denote the set $\left\{\left(G_{x}\right) \mid x \in \mathbb{X}\right\} \subset \mathrm{Orb}_{G}$, that is, the family of orbit types of $\mathbb{X}$.

An equivariant map $f: \mathbb{X} \rightarrow \mathbb{Y}$ is said to be isovariant if $f$ preserves stabilizers, i.e., $G_{x}=G_{f(x)}$ for all $x \in \mathbb{X}$. The category formed by all $G$-spaces and isovariant maps is denoted by ISOV (it is always clear what group $G$ is meant). The following equimorphism criterion is widely known (see [12, Chap. 1, Example 10]).

Proposition 2. An isovariant continuous map is a homeomorphism if and only if the orbit map generated by this map is a homeomorphism. 
Consider a compact group $G$, a metric $H$-space $\mathbb{Y}$, where $H<G$, and the diagonal action

$$
h \cdot(g, y) \rightleftharpoons\left(g \cdot h^{-1}, h \cdot y\right)
$$

of the group $H$ on the product $G \times \mathbb{Y}$. Let $[g, y]$ denote the element

$$
H \cdot(g, y)=\left\{\left(g \cdot h^{-1}, h \cdot y\right) \mid h \in H\right\}
$$

of the orbit space $(G \times \mathbb{Y}) / H$. The relation

$$
g_{1} \cdot[g, y]=\left[g_{1} \cdot g, y\right], \quad \text { where } \quad g, g_{1} \in G, \quad y \in \mathbb{Y},
$$

specifies a well-defined continuous action of $G$ on $(G \times \mathbb{Y}) / H$, which is called the twisted product of $G$ and $\mathbb{Y}$ and denoted by $G \times_{H} \mathbb{Y}$. Any $G$-space $\mathbb{A}$ admitting a $G$-map $\alpha: \mathbb{A} \rightarrow G / H$ to a homogeneous space is equimorphic to the twisted product $G \times_{H} \mathbb{S}$, where $\mathbb{S} \rightleftharpoons \alpha^{-1}([H])$ is an $H$-space, because the continuous $G$-map $\varphi: G \times_{H} \mathbb{S} \rightarrow \mathbb{A}$ defined by $\varphi([g, s])=g \cdot s$ is an equimorphism. We leave the proof of the following fact to the reader.

Lemma 1. Suppose that the $G$-orbit type of a twisted product $G \times_{H} \mathbb{S}$ is single. If the orbit space $\mathbb{S} / H$ is connected, then the $H$-orbit type of $\mathbb{S}$ is single as well.

Below we introduce a number of notions related to the extension of $G$-maps in a category $\mathcal{C}$ coinciding with ISOV or EQUIV. We say that a space $\mathbb{X}$ with an action of a compact group $G$ is an absolute neighborhood $\mathcal{C}$-extensor (and write $\mathbb{X} \in \mathcal{C}$-ANE) if each morphism $\varphi: \mathbb{A} \rightarrow \mathbb{X}$ from $\mathcal{C}$ defined on a closed $G$-subset $\mathbb{A} \subset \mathbb{Z}$ of a $G$-space $\mathbb{Z}$ (which is called a partial $\mathcal{C}$-morphism) can be extended over some $G$-neighborhood $\mathbb{U} \subset \mathbb{Z}$ of the set $\mathbb{A}$ to a morphism $\widehat{\varphi}: \mathbb{U} \rightarrow \mathbb{X} \in \mathcal{C}$. If $\mathbb{U}$ can always be rendered equal to $\mathbb{Z}$, then we say that $\mathbb{X}$ is an absolute $\mathcal{C}$-extensor (and write $\mathbb{X} \in \mathcal{C}$-AE). In the case where the acting group $G$ is trivial (i.e., spaces are considered without actions), this notion transforms into the notion of an absolute [neighborhood] extensor for metric spaces; the class of absolute [neighborhood] extensors is denoted by A[N]E (see [13]).

An absolute [neighborhood] $\mathcal{C}$-extensor is called

- an equivariant [neighborhood] extensor (or, briefly, an Equiv-A[N]E-space) if $\mathcal{C}=$ EQUIV;

- an isovariant [neighborhood] extensor (or, briefly, an Isov-A[N]E-space) if $\mathcal{C}$ coincides with the category ISOV.

Note that the Isov-AE-space coincides with the universal $G$-space in the sense of Palais, and its orbit space classifies $G$-spaces. If there is a $\mathcal{C}$-homotopy $H: \mathbb{X} \times[0,1] \rightarrow \mathbb{Y}$ between morphisms $f, g: \mathbb{X} \rightarrow \mathbb{Y}$ in the category $\mathcal{C}$, then we write

- $f \simeq_{\text {Equiv }} g$ in the case $\mathcal{C}=$ EQUIV,

- $f \simeq_{\text {Isov }} g$ in the case $\mathcal{C}=$ ISOV.

A closed subgroup $H<G$ of a compact group $G$ is called an extensor subgroup if the homogeneous space $G / H$ is a metrizable $G$-ANE-space. As is known, if $H<G$ is an extensor subgroup, then there exists a normal subgroup $P \triangleleft G$ for which (i) $P<H$ and $G / P$ is a compact Lie group; (ii) $G / H$ is a topological manifold; and (iii) $G / H$ is finite-dimensional and locally connected. Each of these three properties characterizes extensor subgroups [14]. The existence of arbitrarily small normal extensor subgroups in any compact group implies the following assertion.

Proposition 3. Given any neighborhood $\mathcal{O}(H) \subset G$ of a subgroup $H$ in a compact group $G$, there exists an extensor subgroup $H^{\prime}<G$ for which $H \subset H^{\prime} \subset \mathcal{O}(H)$.

This and other properties of extensor subgroups are presented in [15], [5]. The well-known notion of a Euclidean neighborhood $G$-retract over a space [7] can be generalized as follows.

MATHEMATICAL NOTES Vol.92 No. 62012 
Definition 1. A $G$-map $f: \mathbb{X} \rightarrow \mathbb{Y}$ is said to be locally equivariantly soft if, for any $G$-diagram

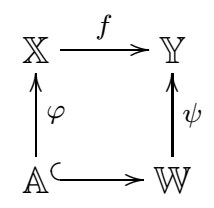

admissible with respect to $f^{1}$, there exists a neighborhood $G$-extension $\widehat{\varphi}: \mathcal{U}(\mathbb{A}) \rightarrow \mathbb{X}$ of $\varphi$ which is a lift of $\psi$.

It is easy to strengthen T. tom Dieck's theorem [7, Sec. 7.6.4.] to the following assertion.

Theorem 6. Suppose that $K<H<G$ and $K<G$ is an extensor subgroup. Then the natural G-map

$$
p: G / K \rightarrow G / H, \quad g \cdot K \mapsto g \cdot H,
$$

is equivariantly locally soft.

An epimorphism $\pi: G \rightarrow H$ of compact groups with kernel $P$ generates the equivalence relation $x \sim x^{\prime} \Leftrightarrow x^{\prime} \in P \cdot x$ on the $G$-space $\mathbb{X}$. Clearly, the quotient space $\mathbb{X} / P$ by this equivalence coincides with $\{P \cdot x \mid x \in \mathbb{X}\}$ and is an $H$-space:

$$
h \cdot(P \cdot x)=P \cdot(g \cdot x), \quad \text { where } \quad g \in \pi^{-1}(h) .
$$

If $y=P \cdot x$, then the stabilizer $H_{y}$ coincides with $\pi\left(G_{x}\right)$.

We refer to the quotient map $f: \mathbb{X} \rightarrow \mathbb{X} / P$ as the $P$-orbit projection. If $P=G$, then $f$ coincides with the orbit projection $p: \mathbb{X} \rightarrow \mathbb{X} / G$. Since the composition of the $P$-orbit projection $f$ with the orbit projection of the $H$-space $(\mathbb{X} / P) / H$ is a perfect map, it follows that this composition itself is a perfect surjection and has the following properties:

$$
\begin{gathered}
f(g x)=\pi(g) \cdot f(x) \quad \text { for all } \quad x \in \mathbb{X}, \quad g \in G, \\
\pi\left(G_{x}\right)=H_{f(x)} \quad \text { for all } \quad x \in \mathbb{X}, \\
\text { if } f(x)=f\left(x^{\prime}\right) \text {, then } x \text { and } x^{\prime} \text { belong to the same } G \text {-orbit. }
\end{gathered}
$$

It turns out that these properties completely characterize $P$-orbit projections [5].

\{eq1:v803

\{eq2:v803

\{eq3:v803

$\{\mathrm{pr} 4: \mathrm{v} 803$

Proposition 4. A perfect surjection $f: \mathbb{X} \rightarrow \mathbb{Y}$ from a $G$-space $\mathbb{X}$ to an $H$-space $\mathbb{Y}$ is a P-orbit projection if and only if $f$ has properties (1)-(3).

Suppose that $K<G, L \rightleftharpoons \pi(K)<H$, and $\pi^{\prime} \rightleftharpoons \pi: K \rightarrow L$ is an epimorphism of compact groups with kernel $Q=P \cap K$. Clearly,

$$
\text { the map } p: G / K \rightarrow H / L, p(g \cdot K)=\pi(g) \cdot L \text {, is a } P \text {-orbit projection. }
$$

\{eq4:v803

Suppose also that $f: \mathbb{X} \rightarrow \mathbb{Y}$ is a $P$-orbit projection and $\varphi: \mathbb{X} \rightarrow G / K$ is the slice map. Clearly, $\mathbb{X}^{\prime} \rightleftharpoons \varphi^{-1}([K])$ is a $K$-space, and $\mathbb{Y}^{\prime} \rightleftharpoons f(\mathbb{X})$ is an $L$-space. Proposition 4 readily implies the following heredity property of orbit projections.

Proposition 5. The restriction map $f^{\prime} \rightleftharpoons f: \mathbb{X}^{\prime} \rightarrow \mathbb{Y}^{\prime}$ is a $Q$-orbit projection.

\footnotetext{
${ }^{1}$ That is, for a $G$-diagram in which $\mathbb{A} \subset \mathbb{W}$ is a closed $G$-subspace, $f, \varphi$, and $\psi$ are $G$-maps, and $f \circ \varphi=\psi \uparrow_{\mathbb{A}}$.
} 
If $K<G$ and $P \backslash K \neq \varnothing$, then there exists an extensor subgroup $\bar{K}<G$ for which $K<\bar{K}<G$ and the map

$$
\bar{p}: G / \bar{K} \rightarrow H / \bar{L}, \quad \bar{p}(g \cdot \bar{K})=\pi(g) \cdot \bar{L}, \quad \text { where } \quad \bar{L} \rightleftharpoons \pi(\bar{K})<H,
$$

is a nontrivial $P$-orbit projection (therefore, by Theorem 6 , it is equivariantly locally soft).

Suppose given an extensor subgroup $K<G$, a $G$-map $f: \mathbb{X} \rightarrow \mathbb{Y}$, a closed $G$-subset $\mathbb{X}_{0} \subset \mathbb{X}$, and a commutative $G$-diagram

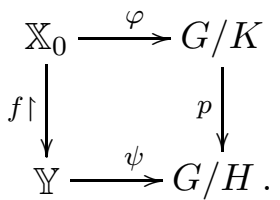

By virtue of Theorem 6, the natural $G$-map $p: G / K \rightarrow G / H$, where $K<H<G$, is equivariantly locally soft, and the commutative $G$-diagram is easy to transform into an admissible $G$-diagram

$$
G / K \stackrel{\varphi}{\longleftarrow} \mathbb{X}_{0} \subset \mathbb{X} \stackrel{\psi \circ f}{\longrightarrow} G / H
$$

with respect to $p$. This readily implies the following theorem, which can be regarded as a generalization of the slice extension theorem for $G$-spaces to the case of $G$-maps.

Theorem 7. There exists a $G$-extension $\widehat{\varphi}: \mathcal{O}\left(\mathbb{X}_{0}\right) \rightarrow G / K$ of the map $\varphi$ to a $G$-neighborhood $\mathcal{O}\left(\mathbb{X}_{0}\right)$ for which $p \circ \widehat{\varphi}=\psi \circ f \uparrow$.

\section{Lie Series for $G$-Spaces}

In a compact group $G$, consider a Lie series $\left\{P_{\alpha} \triangleleft G\right\}$ of normal subgroups indexed by ordinals $\alpha<\tau$ (see [14]). This means that

$$
P_{1}=G, \quad P_{\beta}<P_{\alpha} \text { for all } \alpha<\beta,
$$

$$
P_{\alpha} / P_{\alpha+1} \text { is a compact Lie group for each } \quad \alpha<\tau, \quad \bigcap\left\{P_{\alpha} \mid \alpha<\tau\right\}=\{e\} \text {. }
$$

In this case, $G$ is the $\operatorname{limit} \lim \left\{G / P_{\alpha}, \varphi_{\alpha}^{\beta}\right\}$ of the inverse system of quotient groups $G / P_{\alpha}$ and natural epimorphisms $\chi_{\alpha}^{\beta}: G / P_{\beta} \rightarrow G / P_{\alpha}, \alpha<\beta$. The kernels $\chi_{\alpha}^{\alpha+1}$ of the bonding maps coincide with the compact Lie groups $P_{\alpha} / P_{\alpha+1}$.

Consider a more general construction. Let $\pi_{\alpha}^{\beta}: \mathbb{X}_{\beta} \rightarrow \mathbb{X}_{\alpha}$ be the natural projection of $\mathbb{X}_{\beta}=\mathbb{X} / P_{\beta}$ onto $\mathbb{X}_{\alpha}=\mathbb{X} / P_{\alpha}$. Then $\pi_{\alpha}^{\beta}$ is a $P_{\alpha} / P_{\beta}$-orbit projection, and the map $\pi: \mathbb{X} \rightarrow \lim \left\{\mathbb{X}_{\alpha}, \pi_{\alpha}^{\beta}\right\}$ defined by $\pi(x)=\left\{P_{\alpha} \cdot x\right\}$ is an equimorphism. This assertion is proved by directly applying the equimorphism criterion. Note that the bonding maps $\pi_{\alpha}^{\alpha+1}$ are $P$-orbit projections for the compact Lie group $P=P_{\alpha} / P_{\alpha+1}$. We say that the inverse system $\left\{\mathbb{X}_{\alpha}, \pi_{\alpha}^{\beta}\right\}$ is a Lie series for the $G$-space $\mathbb{X}$.

The converse situation, in which an inverse system is given but the $G$-space is not, often arises. The well-known Theorem 11 from [16, Chap. 2, Sec. 6] can be generalized as follows.

Lemma 2. Let $\left\{P_{\alpha} \triangleleft G\right\}$ be a Lie series. Suppose that, for any $\alpha<\beta, P_{\alpha}^{\beta}$ is the kernel of the homomorphism $\varphi_{\alpha}^{\beta}$ and $g_{\alpha}^{\beta}: \mathbb{Z}_{\beta} \rightarrow \mathbb{Z}_{\alpha}$ is a $P_{\alpha}^{\beta}$-orbit projection; moreover,

$$
g_{\alpha}^{\beta} \circ g_{\beta}^{\gamma}=g_{\alpha}^{\gamma} \quad \text { for all } \quad \alpha<\beta<\gamma .
$$

Then $\mathbb{Z}=\lim _{\longleftarrow}\left\{\mathbb{Z}_{\alpha}, g_{\alpha}^{\beta}\right\}$ is a $G$-space, $\mathbb{Z}_{\alpha}=\mathbb{Z} / P_{\alpha}$, and the inverse system $\left\{\mathbb{Z}_{\alpha}, g_{\alpha}^{\beta}\right\}$ is a Lie series for $\mathbb{Z}$.

The following characterization of $G$-spaces of the form $G / H \times[0,1]$ is, possibly, be known.

Proposition 6. If $\mathbb{Z}=\mathbb{Z}_{(H)}$ and $Z=[0,1]$, then $\mathbb{Z}$ is equimorphic to $G / H \times[0,1]$. 
Proof. The natural projection $\pi: \mathbb{Z}_{H} \rightarrow Z$ can be treated as the orbit projection under the action of the normalizer $N(H)$ on $\mathbb{Z}_{H}$; i.e., we can assume that $Z=\mathbb{Z}_{H} / N(H)$. According to Proposition 7 , the map $\pi$ has a section $\sigma: Z \rightarrow \mathbb{Z}_{H}$. Thus,

$$
\varphi: G / H \times[0,1] \rightarrow \mathbb{Z}, \quad \varphi(g H, t)=g \cdot \sigma(t),
$$

is a well-defined equimorphism.

Proposition 7. If a compact group $G$ acts on a space $\mathbb{X}$ of a single orbit type, then the orbit projection $p: \mathbb{X} \rightarrow X$ is a Hurewicz bundle.

Proof. Consider the expansion of the $G$-space $\mathbb{X}$ in a Lie series $\left\{\mathbb{X}_{\alpha}, \pi_{\alpha}^{\beta}\right\}$. Since $P=P_{\alpha} / P_{\alpha+1}$ is a compact Lie group, it follows by Lemma 3 that the bonding $P$-orbit projections $\pi_{\alpha}^{\alpha+1}$ are locally trivial bundles and, therefore, Hurewicz bundles.

Lemma 3. Any P-orbit projection $f: \mathbb{X} \rightarrow \mathbb{Y}$ from a $G$-space $\mathbb{X}$ of a single orbit type to an $H$-space $\mathbb{Y}$, where the kernel $P$ of the epimorphism $\pi: G \rightarrow H$ is a compact Lie group, is a locally trivial bundle.

Proof of Lemma 3. The required assertion follows readily from the slice extension theorem.

\section{ISOVARIANT EXTENSORS}

Obviously, Theorem 1 is implied by the following more precise theorem.

Theorem 8. Suppose that, for any $i \geq 1, \mathbb{X}_{i}$ is an Isov-generating Equiv-AE-space. Then,

$$
\begin{aligned}
& \text { for any partial } G \text {-map } \mathbb{Z} \hookleftarrow \mathbb{A} \stackrel{\varphi}{\rightarrow} \rightleftharpoons \prod\left\{\mathbb{X}_{i} \mid i \geq 1\right\}, \\
& \text { there exists a } G \text {-map } \psi: \mathbb{Z} \rightarrow \mathbb{X} \text { extending } \varphi \\
& \text { for which } \psi \uparrow_{\mathbb{Z} \backslash \mathbb{A}} \text { is an isovariant map. }
\end{aligned}
$$

$\{\sec 2: \mathrm{v} 80$

$\{$ th8: 8803

Proof. Since $\mathbb{X} \in$ Equiv-AE, it is sufficient to find, given any equivariant map $\widehat{\varphi}: \mathbb{Z} \rightarrow \mathbb{X}$, a $G$-map $\psi: \mathbb{Z} \rightarrow \mathbb{X}$ extending $\varphi=\widehat{\varphi} \Upsilon_{\mathbb{A}}$ for which $\psi \uparrow_{\mathbb{Z} \backslash \mathbb{A}}$ is an isovariant map. Since $\mathbb{A} \subset \mathbb{X}$ is closed, we can choose a sequence of neighborhoods $\mathbb{Z}=\mathbb{U}_{0} \ni \mathbb{U}_{1} \ni \cdots$ and $G$-functions $\chi_{i}: \mathbb{Z} \rightarrow[0,1], i \geq 1$, so that

$$
\bigcap \mathbb{U}_{i}=\mathbb{A}, \quad \chi_{i}^{-1}(0) \supset \mathbb{Z} \backslash \mathbb{U}_{i}, \quad \chi_{i}^{-1}(1) \supset \mathbb{U}_{i+1}^{2} .
$$

Let us represent the map $\widehat{\varphi}$ in the form $\prod \widehat{\varphi}_{i}$, where $\widehat{\varphi}_{i}: \mathbb{Z} \rightarrow \mathbb{X}_{i}$ is an equivariant map. Take an isovariant map $e_{i}: \mathbb{Z} \rightarrow \mathbb{X}_{i}$ (which exists, because $\mathbb{X}_{i}$ is Isov-generating) and let $H_{i}: \mathbb{Z} \times I \rightarrow \mathbb{X}_{i}$ be an Equiv-homotopy between $e_{i}$ and $\widehat{\varphi}_{i}$ (which exists as well, because $\mathbb{X}_{i} \in$ Equiv-AE). Then the required map $\psi$ is defined by

$$
\begin{gathered}
\psi \Upsilon_{\mathbb{A}}=\widehat{\varphi} \Upsilon_{\mathbb{A}}=\varphi \\
\left(\psi \Upsilon_{\mathbb{U}_{i} \backslash \mathbb{U}_{i+1}}\right)(z)=\widehat{\varphi}_{1} \times \cdots \times \widehat{\varphi}_{i-1} \times H_{i}\left(z, \chi_{i}(z)\right) \times e_{i+1} \times \cdots \quad \text { for } \quad i \geq 0 .
\end{gathered}
$$

Take a closed topological embedding $j: X \hookrightarrow L$ of the orbit space of any $G$-space $\mathbb{X}$ into some normed linear space $L[17]$. Since the countable power $\mathbb{J}$ of the metric cone Con $\mathbb{T}$ over

$$
\mathbb{T}=\bigsqcup\{G / H \mid G / H \in G \text {-ANE }\}
$$

is Isov-generating [5], it follows that there exists an isovariant map $f: \mathbb{X} \rightarrow \mathbb{J}$. Obviously, the product $(j \circ p) \times f$ is a closed topological $G$-embedding of $\mathbb{X}$ into the $G$-space $\mathbb{Y} \rightleftharpoons L \times \mathbb{J}$; obviously, this $G$ space is an Isov-AE. Thus, we have proved the following theorem.

Theorem 9. Any G-space admits a closed $G$-embedding into an Isov-AE-space of the form $L \times \mathbb{J}$.

\footnotetext{
${ }^{2}$ We say that an embedding $A \subset B$ is strict and write $A \Subset B$ if $\mathrm{Cl} A \subset \operatorname{Int} B$.
} 
An easy consequence of Theorems 9 and 8 is an important relation between the injective objects of the isovariant and the equivariant category.

Theorem 10. Any Isov-A[N]E-space $\mathbb{X}$ has property (6); therefore, any Isov-A[N]E-space is an Equiv-A[N]E-space.

We mention without proof that the equivariant Hilbert cube $\mathbb{Q}$ (for a compact metric group $G$ ) is an Isov-AE. On the other hand, if $\mathbb{X}$ is a compact Isov-AE-space, then the product of $\mathbb{X}$ and the Hilbert cube $Q$ is equimorphic to $\mathbb{Q}$. Other examples of Isov-AE-spaces are the equivariant space $\mathbb{L}_{2}$ and the space $\mathrm{C}(G, L)$ (with the metric of uniform convergence) of all continuous maps $f: G \rightarrow L$ to an equivariant Hilbert space $L$ of weight $w(G)$ on with the group $G$ continuously acts by the rule

$$
(g \cdot f)(h)=f\left(g^{-1} \cdot h\right), \quad \text { where } f \in \mathrm{C}(G, L), \quad g, h \in G .
$$

These and other results of the theory of isovariant extensors will be published elsewhere.

It is easy to give an example of an Equiv-AE-space $\mathbb{Z} \notin$ Isov-ANE. In what follows, we obtain several results which make it possible to assess the degree of dissimilarity between these two classes; namely, $\mathbb{X} \in$ Equiv-ANE implies the equi-local contractibility (equi-LC) property for the family $\left\{\mathbb{X}^{H} \mid H<G\right\}$, and $\mathbb{X} \in$ Isov-ANE implies $\left\{\mathbb{X}_{H} \mid H<G\right\} \in$ equi-LC; moreover, Con $\mathbb{X} \in$ Isov-AE and $\mathbb{X}^{G} \in \mathrm{AE}$ imply $\mathbb{X} \in \mathrm{Isov}$-AE.

Apparently, the following questions are of certain interest in relation to the Hilbert-Smith conjecture [18]. Suppose that the acting group $G$ is nontrivial and $\mathbb{X} \in$ Isov-AE. Is it true that $\operatorname{dim} \mathbb{X}=\infty$ ? $\operatorname{dim} X=\infty$ ?

\section{JOINING SPLITTINGS}

The restriction of an admissible diagram $\mathcal{D}$ to an invariant subset $\mathbb{U} \subset \mathbb{T}$ is defined as the diagram

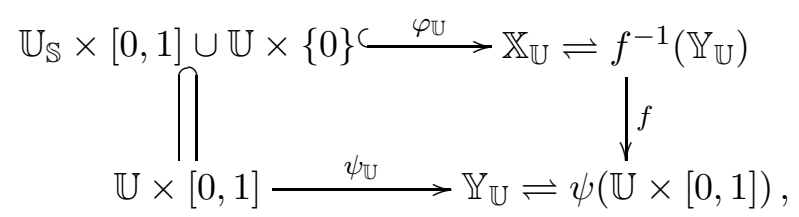

denoted by $\mathcal{D}_{\mathbb{U}}$, in which $\varphi_{\mathbb{U}}$ is the restriction of $\varphi$ to $\mathbb{U}_{\mathbb{S}} \times I \cup \mathbb{U} \times\{0\}, \psi_{\mathbb{U}} \rightleftharpoons \psi \uparrow_{\mathbb{U} \times I}$, and $\mathbb{U}_{\mathbb{S}} \rightleftharpoons \mathbb{U} \cap \mathbb{S}$. Clearly, $\mathcal{D}_{\mathbb{U}}$ is an admissible diagram; if the diagram $\mathcal{D}$ splits, then so does $\mathcal{D}_{\mathbb{U}}$.

The following proposition on joining splittings is a modification of an argument used by Palais in [1] to a more general situation.

Proposition 8. Let $\left\{\mathbb{T}_{\lambda} \mid \lambda \in \Lambda\right\}$ be an open locally finite $G$-cover of $\mathbb{T}$ such that the restriction $\mathcal{D}_{\lambda}$ of an admissible diagram $\mathcal{D}$ to $\mathbb{T}_{\lambda}$ splits for any $\lambda \in \Lambda$. Then the diagram $\mathcal{D}$ splits as well.

First, consider the case of a two-element index set $\Lambda$.

Lemma 4 (on joining two splittings). Let $\left\{\mathbb{T}_{1}, \mathbb{T}_{2}\right\}$ be an open $G$-cover of $\mathbb{T}$, and let $\mathcal{D}_{i}$ be the restriction of an admissible diagram $\mathcal{D}$ to $\mathbb{T}_{i}$. If G-homeomorphisms $g_{i}: \mathbb{T}_{i} \times[0,1] \rightarrow \mathbb{X}_{\mathbb{T}_{i}}, i \leq 2$, split the diagrams $(\mathcal{D})_{i}$, respectively, then there exists a G-homeomorphism $g: \mathbb{T} \times[0,1] \rightarrow \mathbb{X}$ splitting the diagram $\mathcal{D}$. Moreover,

$$
g=g_{i} \quad \text { on } \quad \mathbb{T}_{i} \backslash \mathbb{T}_{0}, \quad \text { where } \quad \mathbb{T}_{0} \rightleftharpoons \mathbb{T}_{1} \cap \mathbb{T}_{2} .
$$

Proof. The proof of Lemma 4 is based on the following homotopy splitting effect. 
Lemma 5 (on homotopy splitting). If the G-homeomorphisms $\widehat{\varphi}_{i}: \mathbb{T} \times[0,1] \rightarrow \mathbb{X}, i=1,2$, split the admissible diagram $\mathcal{D}$, then there exist isovariant $G$-homotopies

$$
H_{s}: \mathbb{T} \times[0,1] \rightarrow \mathbb{X}, \quad 0 \leq s \leq 1,
$$

between $\widehat{\varphi}_{1}$ and $\widehat{\varphi}_{2}$ such that the $H_{s}$ split the diagram $\mathcal{D}^{3}$ for all $0 \leq s \leq 1$.

(The proof of Lemma 5 is given after the proof of Proposition 8.)

According to Lemma 5 on homotopy splitting, there exist

isovariant $G$-homotopies $H_{s}: \mathbb{T}_{0} \times[0,1] \rightarrow \mathbb{X}_{\mathbb{T}_{0}}, 0 \leq s \leq 1$, between $g_{1} \uparrow_{\mathbb{T}_{0}}$ and $g_{2} \uparrow_{\mathbb{T}_{0}}$

such that the $G$-homeomorphisms $H_{s}$ split the diagram $\mathcal{D}_{\mathbb{T}_{0}}$ for all $0 \leq s \leq 1$.

Since $\mathbb{T} \backslash \mathbb{T}_{2}$ and $\mathbb{T} \backslash \mathbb{T}_{1}$ are disjoint closed subsets of the normal space $\mathbb{T}$, it follows that there exists an invariant function $\xi: \mathbb{T} \rightarrow[1,2]$ such that

$$
\begin{array}{lll}
\xi \equiv 0 & \text { in a neighborhood of } & \mathbb{T} \backslash \mathbb{T}_{2}, \\
\xi \equiv 1 & \text { in a neighborhood of } & \mathbb{T} \backslash \mathbb{T}_{1} .
\end{array}
$$

Finally, we set

$$
g(u, t)= \begin{cases}g_{1}(u, t) & \text { if }(u, t) \in\left(T \backslash T_{2}\right) \times I, \\ H_{\xi(u)}(u, t) & \text { if }(u, t) \in T_{0} \times I, \\ g_{2}(u, t) & \text { if }(u, t) \in\left(T \backslash T_{1}\right) \times I .\end{cases}
$$

The continuity of the resulting map $g: \mathbb{T} \times I \rightarrow \mathbb{Z}$ is checked directly by using (9). It follows from (8) that $g$ splits the diagram $\mathcal{D}$. This completes the proof of Lemma 4.

The rest of the proof of Proposition 8 on joining splittings proceeds by standard transfinite induction on the well-ordered index set $\Lambda$ by using Lemma 4 on joining two splittings. The stabilization of the map $g$ (to be constructed) which splits the diagram $\mathcal{D}$ and its continuity follow from the local finiteness of the cover $\left\{\mathbb{T}_{\lambda}\right\}$ and property ( 7$)$. We leave the details to the reader.

Proof of Lemma 5. It follows readily by assumption that $h \rightleftharpoons\left(\widehat{\varphi}_{1}\right)^{-1} \circ \widehat{\varphi}_{2}$ is a $G$-self-homeomorphism of $\mathbb{T} \times[0,1]$ which is the identity map on $\mathbb{S} \times[0,1] \cup \mathbb{T} \times\{0\}$. Since

$$
\psi=p \circ \widehat{\varphi}_{2}=\left(\psi \circ \widehat{\varphi}_{1}^{-1}\right) \circ \widehat{\varphi}_{2}=\psi \circ h,
$$

it follows that $h$ has the form

$$
h(z, t)=\left(\varphi_{t}(z), t\right)
$$

where $\varphi_{t}: \mathbb{T} \rightarrow \mathbb{T}$ is a continuous (in $t \in T$ ) family of $G$-homeomorphisms. Clearly,

$$
\varphi_{0}=\mathrm{Id}, \quad \varphi_{t} \Gamma_{\mathbb{S}}=\mathrm{Id} \quad \text { for all } t \in I \text {. }
$$

By virtue of (10), we have

$$
\psi(w)=\psi(P \cdot w)=\psi(h(P \cdot w)) \quad \text { for all } \quad w=(z, t) \in \mathbb{T} \times I ;
$$

hence

$$
\left(\psi_{P}\right)(P \cdot z, t)=\left(\psi_{P}\right)\left(P \cdot \varphi_{t}(z), t\right) .
$$

Taking into account the fact that $\psi_{P}$ is an $H$-homeomorphism, we obtain

$$
P \cdot z=P \cdot \varphi_{t}(z) \quad \text { for all } \quad t \in I .
$$

Now, we set

$$
h_{s}(u, t)=\left(\varphi_{s \cdot t}(u), t\right): \mathbb{T} \times I \rightarrow \mathbb{T} \times I, \quad 0 \leq s \leq 1 .
$$

\footnotetext{
${ }^{3}$ Therefore, $H_{s}$ is an $G$-homeomorphism.
} 
It can be verified directly that $h_{s}$ is a continuous family of $G$-homeomorphisms, and, moreover,

$$
h_{1}=h, \quad h_{0}=\mathrm{Id}, \quad h_{s}=h \quad \text { on } \quad \mathbb{S} \times[0,1] \cup \mathbb{T} \times\{0\} .
$$

By using (11), it is easy to derive that

$$
\psi=\psi \circ h_{s} \quad \text { for all } s \in I .
$$

Finally, we define the required isovariant homotopy by

$$
H_{s} \rightleftharpoons \widehat{\varphi}_{1} \circ h_{s}, \quad 0 \leq s \leq 1 .
$$

All of the required properties of $H_{s}$ follow from (12) and (13).

\section{PROOF OF THEOREM 3}

It suffices to prove that the $P$-orbit projection $f: \mathbb{X} \rightarrow \mathbb{Y}=\mathbb{X} / P$ is a Hurewicz Isov-bundle. Indeed, if there is an admissible diagram $\mathcal{D}$ for the $P$-orbit projection $f$ from Theorem 3 , then we pass from $\mathbb{Y}$ to the image $\mathbb{Y}^{\prime} \subset(T \times I) \times \mathbb{Y}$ of $\mathbb{T} \times I$ under the $G$-map

$$
q \times \psi: \mathbb{T} \times I \rightarrow(T \times I) \times \mathbb{Y}
$$

and from $\mathbb{X}$ to the preimage $\mathbb{X}^{\prime} \subset(T \times I) \times \mathbb{X}$ of $\mathbb{Y}^{\prime}$ under the $P$-orbit projection

$$
f^{\prime}=\operatorname{Id} \times f:(T \times I) \times \mathbb{X} \rightarrow(T \times I) \times \mathbb{Y}
$$

(here $q: \mathbb{T} \times I \rightarrow T \times I$ is the orbit projection). Let $\varphi^{\prime}$ and $\psi^{\prime}$ denote the maps

$$
q \times \varphi: \mathbb{S} \times[0,1] \cup \mathbb{T} \times\{0\} \rightarrow \mathbb{X}^{\prime}, \quad q \times \psi: \mathbb{T} \times[0,1] \rightarrow \mathbb{Y}^{\prime},
$$

respectively. Clearly, the $P$-orbit projection $f^{\prime}: \mathbb{X}^{\prime} \rightarrow \mathbb{Y}^{\prime}$ is naturally involved in an admissible diagram $\mathcal{D}^{\prime}$, which is weakly admissible, because $\psi^{\prime}$ induces a homeomorphism of the orbit spaces. The validity of Theorem 3 for weakly admissible diagrams implies the existence of an isovariant $G$-map $\widehat{\varphi}^{\prime}: \mathbb{T} \times[0,1] \rightarrow \mathbb{X}^{\prime}$ which splits $\mathcal{D}^{\prime}$. The required isovariant $G$-map $\widehat{\varphi}: \mathbb{T} \times[0,1] \rightarrow \mathbb{X}$ splitting $\mathcal{D}$ is the composition of $\hat{\varphi}^{\prime}$ with the projection $(T \times I) \times \mathbb{X}$ onto the factor $\mathbb{X}$.

In any completely admissible diagram $\mathcal{D}$, the isovariant map

$$
\varphi: \mathbb{S} \times[0,1] \cup \mathbb{T} \times\{0\} \rightarrow \mathbb{X}
$$

is a closed $G$-embedding. Thus, in the proof of Theorem 3, we can assume without loss of generality that

1) $\mathbb{S} \times[0,1] \cup \mathbb{T} \times\{0\}$ is a closed subset of $\mathbb{X}$, and $\varphi$ is the identity embedding;

2) $\mathbb{Y}=(\mathbb{T} / P) \times I$, and $\psi_{P}:(\mathbb{T} / P) \times I \rightarrow \mathbb{Y}$ is the identity map;

3) the map $f \uparrow_{\mathbb{S} \times[0,1] \cup \mathbb{T} \times\{0\}}$ coincides with the restriction of the canonical $P$-orbit projection $\mathbb{T} \times$ $[0,1] \rightarrow T \times[0,1]$ to $\mathbb{S} \times[0,1] \cup \mathbb{T} \times\{0\}$.

The rest of the proof of Theorem 3 asserting that the weakly admissible $G$-diagram is split proceeds by induction on the compact Lie group $P$ by using Palais' metatheorem [1], whose proof is based on the stabilization of a decreasing (by inclusion) sequence of compact Lie groups.

Proposition 9. Let $\mathcal{P}(H)$ be a property depending on a compact Lie group $H$. Suppose that $\mathcal{P}(H)$ holds for the trivial group $H=\{e\}$ and $\mathcal{P}(H)$ holds if so does $\mathcal{P}(K)$ for any proper subgroup $K<H$. Then $\mathcal{P}(H)$ holds for all compact Lie groups $H$.

If $|P|=1$, then the epimorphism $\pi: G \rightarrow H$ is an isomorphism, which trivializes the situation under examination. Suppose that, for any proper subgroup $Q<P$, Theorem 3 is valid. Let us show that it is valid for any $P$-orbit projection $f: \mathbb{X} \rightarrow \mathbb{Y}$.

The following lemma reduces proving that the weakly admissible diagram $\mathcal{D}$ splits to the case in which there are no $P$-fixed points in $\mathbb{X}$. 
Lemma 6. If any weakly admissible diagram $\mathcal{D}$ with empty $\mathbb{X}^{P}$ splits, then so does any weakly admissible diagram.

Proof. By assumption, there exists an isovariant map

$$
\varphi^{\prime}:\left(\mathbb{T} \backslash \mathbb{T}^{P}\right) \times I \rightarrow \mathbb{X} \backslash \mathbb{X}^{P}
$$

splitting the weakly admissible diagram $\mathcal{D}_{\mathbb{T} \backslash \mathbb{T}^{P}}$. We extend the map $\varphi^{\prime}$ so that the extension coincides with $\psi$ on $\mathbb{T}^{P} \times I$. It is easy to check that $\varphi^{\prime}$ is well defined and continuous.

Let $\mathcal{D}$ be a weakly admissible diagram. By virtue of Lemma 6 , in what follows, we can assume that $\mathbb{X}^{P}=\varnothing$ for the $G$-space $\mathbb{X}$ (or, equivalently, that $P \backslash G_{x} \neq \varnothing$ for all $x \in \mathbb{X}$ ). The product

$$
\mathbb{S} \times[0,1] \cup \mathbb{T} \times\{0\}
$$

is naturally embedded in $\mathbb{X}$; therefore, $\mathbb{T}^{P}=\varnothing$.

First, suppose that $\mathbb{T}$ admits a nontrivial slice map $\alpha: \mathbb{T} \rightarrow G / K$, where $K<G$ is an extensor subgroup with $P \backslash K \neq \varnothing$. Then the $G$-map

$$
\beta \rightleftharpoons \alpha \circ \operatorname{pr}_{1}: \mathbb{T} \times[0,1] \rightarrow G / K,
$$

where $\operatorname{pr}_{1}: \mathbb{T} \times I \rightarrow \mathbb{T}$ is the projection onto the first factor, generates the slice map

$$
\beta_{P}: \mathbb{Y}=(\mathbb{T} / P) \times[0,1] \rightarrow G /(P \cdot K)=H / L, \quad \text { where } \quad L \rightleftharpoons \pi(K)<H,
$$

under the passage to the $P$-orbit projection. Let

$$
p: G / K \rightarrow G / \pi^{-1}(\pi(K)) \cong H / L
$$

denote the natural $G$-map, which is a $P$-orbit projection.

Lemma 7. If the partial G-map

$$
\mathbb{X} \hookleftarrow \mathbb{S} \times[0,1] \cup \mathbb{T} \times\{0\} \stackrel{\beta \uparrow}{\longrightarrow} G / K
$$

admits a G-extension $\widehat{\beta}: \mathbb{X} \rightarrow G / K$ for which $\beta_{P} \circ f=p \circ \widehat{\beta}$, then the diagram $\mathcal{D}$ splits.

Proof. Let $\pi^{\prime} \rightleftharpoons \pi \uparrow: K \rightarrow L$ be the epimorphism of compact groups with kernel $Q=P \cap K$. Since $P \backslash K \neq \varnothing$, it follows that $Q$ is a proper subgroup in the Lie group $P$.

Now consider the $K$-space $\mathbb{T}^{\prime} \rightleftharpoons \alpha^{-1}([K])$, its closed subspace $\mathbb{S}^{\prime} \rightleftharpoons \mathbb{T}^{\prime} \cap \mathbb{S}$, and the $K$-space $\mathbb{X}^{\prime} \rightleftharpoons \widehat{\beta}^{-1}([K])$. Clearly, the $L$-space $\mathbb{Y}^{\prime} \rightleftharpoons \beta_{P}^{-1}([L])$ coincides with $f\left(\mathbb{X}^{\prime}\right)$. It follows from Proposition 5 on the heredity of orbit projections that the restriction map $f^{\prime}=f \uparrow: \mathbb{X}^{\prime} \rightarrow \mathbb{Y}^{\prime}$ is a $Q$-orbit projection.

We have $\widehat{\beta}=\operatorname{ext}\left(\beta \uparrow_{\mathbb{S} \times[0,1] \cup \mathbb{T} \times\{0\}}\right)$; hence

$$
\varphi\left(\mathbb{S}^{\prime} \times I \cup \mathbb{T}^{\prime} \times\{0\}\right) \subset \mathbb{X}^{\prime}, \quad \psi\left(\mathbb{T}^{\prime} \times I\right)=\mathbb{Y}^{\prime}
$$

Let

$$
\varphi^{\prime}: \mathbb{S}^{\prime} \times I \cup \mathbb{T}^{\prime} \times\{0\} \hookrightarrow \mathbb{X}^{\prime} \quad \psi^{\prime}: \mathbb{T}^{\prime} \times I \rightarrow \mathbb{Y}^{\prime}
$$

be the restrictions of $\varphi$ and $\psi$ to the corresponding subsets. Consider the naturally arising commutative $K$-diagram

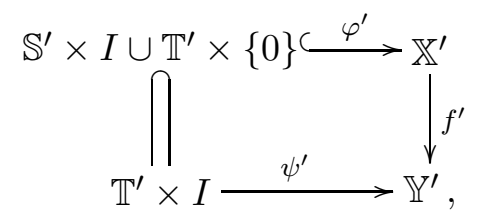


in which $\psi^{\prime}$ induces a homeomorphism of the orbit spaces and $\varphi^{\prime}$ is a $K$-embedding; we denote this diagram by $\mathcal{D}^{\prime}$. It follows readily from Lemma 1 that the $G$-space $\mathbb{Z} \rightleftharpoons\left(f^{\prime}\right)^{-1}\left(\psi^{\prime}\left(t^{\prime} \times I\right)\right)$ is of a single orbit type; therefore, the commutative $K$-diagram $\mathcal{D}^{\prime}$ is weakly admissible.

The subgroup $Q<P$ is proper. Therefore, by the induction hypothesis, there exists a $K$-map $\widehat{\varphi}^{\prime}: \mathbb{T}^{\prime} \times I \rightarrow \mathbb{X}^{\prime}$ splitting $\mathcal{D}^{\prime}$. A direct verification shows that the rule

$$
\widehat{\varphi}\left([g, s]_{K}\right)=\left[g, \hat{\varphi}^{\prime}(s)\right]_{K}, \quad s \in \mathbb{T}^{\prime} \times I
$$

well defines a $G$-map

$$
\widehat{\varphi}: G \times_{K}\left(\mathbb{T}^{\prime} \times I\right) \equiv \mathbb{T} \times I \rightarrow G \times_{K} \mathbb{X}^{\prime} \equiv \mathbb{X}
$$

which splits the diagram $\mathcal{D}$.

Let us show that the assumptions of Lemma 7 (and, hence, its conclusions) hold for the elements of some invariant cover of $\mathbb{T}$. By virtue of the splitting joining theorem and the induction hypothesis, Theorem 3 will follow.

Take any point $t \in \mathbb{T}$. The relation $P \backslash G_{t} \neq \varnothing$ and Proposition 3 imply the existence of an extensor subgroup $K<G$ for which $G_{t}<K$ and $P \backslash K \neq \varnothing$. Since $G / K \in G$-ANE, it follows that there exists a slice map $\alpha: \mathbb{U} \rightarrow G / K$, where $\mathbb{U}=\mathbb{U}(t) \subset \mathbb{T}$ is a $G$-neighborhood of $t$.

Let $L \rightleftharpoons \pi(K)<H$, and let

$$
p: G / K \rightarrow G / \pi^{-1}(\pi(K)) \cong H / L
$$

be the natural $G$-map generated the embedding of groups $K<\pi^{-1}(\pi(K))$. Consider the slice map

$$
\left.\beta_{P}: \psi(\mathbb{U}) \times[0,1]\right)=(\mathbb{U} / P) \times[0,1] \rightarrow G /(P \cdot K)=H / L
$$

obtained from the $G$-map $\beta \rightleftharpoons \alpha \circ \operatorname{pr}_{1}: \mathbb{U} \times[0,1] \rightarrow G / K$ under the passage to the $P$-orbit projection.

Proposition 10. There exists a $G$-neighborhood $\mathbb{V} \subset \mathbb{U}$ of the point $t$ for which the partial $G$-map

$$
\left(f^{-1} \circ \psi\right)(\mathbb{V} \times I) \hookleftarrow(\mathbb{S} \cap \mathbb{V}) \times I \cup \mathbb{V} \times\{0\} \stackrel{\beta}{\rightarrow} G / K
$$

admits a G-extension

$$
\widehat{\beta}:\left(f^{-1} \circ \psi\right)(\mathbb{V} \times I) \rightarrow G / K \quad \text { for which } \quad \beta_{P} \circ f=p \circ \widehat{\beta} .
$$

Proof. Without loss of generality, we can assume that $\mathbb{U}$ coincides with $\mathbb{T}$, i.e., there exists a slice map $\alpha: \mathbb{T} \rightarrow G / K$.

Consider the $G$-space $\mathbb{A} \rightleftharpoons f^{-1}(\psi(\{t\} \times I)) \subset \mathbb{X}$. Clearly,

$$
\mathbb{A} \subset \mathbb{S} \times I \quad \text { for } t \in \mathbb{S} \quad \text { and } \quad \mathbb{A} \cap(\mathbb{S} \times I \cup \mathbb{T} \times\{0\})=G(t) \times\{0\} \quad \text { for } t \notin \mathbb{S} .
$$

Note that $\mathbb{A}$ has single orbit type $\left(G_{t}\right)$, and its orbit space is homeomorphic to $I$. Consequently, by virtue of Proposition 6, $\mathbb{A}$ is equimorphic to $G(t) \times I$. Hence there exists a slice map

$$
\gamma: \mathbb{S} \times I \cup \mathbb{T} \times\{0\} \cup \mathbb{A} \rightarrow G / K
$$

on $\mathbb{S} \times I \cup \mathbb{T} \times\{0\} \cup \mathbb{A}$ which coincides with $\beta$ on $\mathbb{S} \times I \cup \mathbb{T} \times\{0\}$ and satisfies the condition $\beta_{P} \circ f=$ $p \circ \gamma$.

According to Theorem 6 , the natural $G$-map $p: G / K \rightarrow G / \pi^{-1}(\pi(K))$ is equivariantly locally soft, and according to Theorem 7 , there exists a $G$-extension $\widehat{\beta}: \mathbb{W} \rightarrow G / K$ of $\gamma$ to a $G$-neighborhood

$$
\mathbb{W} \supset \mathbb{S} \times I \cup \mathbb{T} \times\{0\} \cup \mathbb{A}
$$

for which $\beta_{P} \circ f=p \circ \widehat{\beta}$. Reducing, if necessary, the neighborhood $\mathbb{W}$ to a neighborhood of the form $\left(f^{-1} \circ \psi\right)(\mathbb{V} \times I)$, we obtain the required slice map $\widehat{\beta}$. 


\section{ACKNOWLEDGMENTS}

The first author acknowledges the support of the Ministry of education of the Republic of Belarus, while the second author acknowledges the support of the Slovenian Research Agency (projects nos. P1-0292-0101, J1-9643-0101, and J1-2057-0101).

\section{REFERENCES}

1. R. S. Palais, The Classification of G-Spaces, in Mem. Amer. Math. Soc. (Amer. Math. Soc., Providence, RI, 1960), Vol. 36.

2. S. M. Ageev, "Classification of $G$-spaces," Izv. Ross. Akad. Nauk Ser. Mat. 56 (6), 1345-1357 (1992) [Russian Acad. Sci. Izv. Math. 41 (3), 581-591 (1993)].

3. S. M. Ageev, "Isovariant extensors," Sibirsk. Mat. Zhurn. [Siberian Math. J.](in press).

4. S. M. Ageev, "Extensor properties of orbit spaces and the problem of the continuation of an action," Vestnik Moskov. Univ. Ser. I Mat. Mekh., No. 1, 11-16 (1994) [Moscow Univ. Math. Bull. 49 (1), 9-12 (1995)].

5. S. M. Ageev and D. Repovš, "On the extension of actions of groups," Mat. Sb. 201 (2), 3-28 (2010) [Russian Acad. Sci. Sb. Math. $201(1-2), 159-182(2010)]$.

6. T. tom Dieck, Transformation Groups, in de Gruyter Stud. Math. (Walter de Gruyter, Berlin, 1987), Vol. 8.

7. T. tom Dieck, Transformations Groups and Representation Theory, in Lecture Notes in Mathematics (Springer-Verlag, Berlin-Heidelberg-New York, 1979; Mir, Moscow, 1982), Vol. 766.

8. S. Waner, "A generalization of the cohomology of groups," Proc. Amer. Math. Soc. 85 (3), 469-474 (1982).

9. S. Waner, "Mackey functors and $G$-cohomology," Proc. Amer. Math. Soc. 90 (4), 641-648.

10. J. P. May, Equivariant Homotopy and Cohomology Theory, in CBMS Regional Conf. Ser. in Math. (Amer. Math. Soc., Providence, RI, 1996), Vol. 91.

11. S. M. Ageev, "Isovariant extensors and the characterization of equivariant homotopy equivalences," Izv. Ross. Akad. Nauk Ser. Mat. 76 (5), 3-28 (2012) [Russian Acad. Sci. Izv. Math. 76 (5), 857-880 (2012)].

12. G. E. Bredon, Introduction to Compact Transformation Groups, in Pure and Applied Mathematics (Academic, New York-London, 1972; (Nauka, Moscow, 1980), VOl. 46.

13. K. Borsuk, Theory of Retracts (PWN, Warzsawa, 1967; Mir, Moscow, 1971).

14. L. S. Pontryagin, Continuous Groups (Nauka, Moscow, 1984) [in Russian].

15. S. M. Ageev, "An equivariant Dugundji theorem," Uspekhi Mat. Nauk 45 (5), 179-180 (1990) [Russian Math. Surveys 45 (5), 219-220 (1990)].

16. E. H. Spanier, Algebraic Topology (McGraw-Hill, New York, 1966; Mir, Moscow, 1971).

17. S.-T. Hu, Theory of Retracts (Wayne State University Press, Detroit, 1965).

18. S. M. Ageev, "Classifying spaces for free actions and the Hilbert-Smith conjecture," Mat. Sb. 183 (1), 143151 (1992) [Russian Acad. Sci. Sb. Math. 75 (1), 137-144 (1993)]. 